Incidence rates, correlates, and prognosis of electrocardiographic P-wave abnormalities - a nationwide population-based study

\author{
Lehtonen, Arttu O.
}

2017

Lehtonen, A O , Langen, V L , Puukka, P J , Kahonen, M , Nieminen , M S , Jula , A M \& Niiranen, T J 2017 , ' Incidence rates, correlates, and prognosis of electrocardiographic P-wave abnormalities - a nationwide population-based study ' , Journal of Electrocardiology , vol. 50 , no. 6 , pp. 925-932 . https://doi.org/10.1016/j.jelectrocard.2017.07.004

http://hdl.handle.net/10138/298172

https://doi.org/10.1016/j.jelectrocard.2017.07.004

publishedVersion

Downloaded from Helda, University of Helsinki institutional repository.

This is an electronic reprint of the original article.

This reprint may differ from the original in pagination and typographic detail.

Please cite the original version. 


\title{
Incidence rates, correlates, and prognosis of electrocardiographic P-wave abnormalities - a nationwide population-based study
}

\author{
Arttu O. Lehtonen, MD, ${ }^{a}, \mathrm{~b}, *$ Ville L. Langén, MD, ${ }^{\mathrm{a}, \mathrm{c}, \mathrm{d}}$ Pauli J. Puukka, MSocSc, ${ }^{\mathrm{a}}$ \\ Mika Kähönen, $\mathrm{MD}, \mathrm{PhD},{ }^{\mathrm{e}}$ Markku S. Nieminen, $\mathrm{MD}, \mathrm{PhD},{ }^{\mathrm{f}}$ Antti M. Jula, MD, $\mathrm{PhD},{ }^{\mathrm{a}, \mathrm{d}}$ \\ Teemu J. Niiranen, $\mathrm{MD}, \mathrm{PhD}^{\mathrm{a}, \mathrm{g}}$ \\ ${ }^{a}$ Department of Health, National Institute for Health and Welfare, Turku, Finland \\ ${ }^{\mathrm{b}}$ Department of Geriatrics, Turku City Hospital and University of Turku, Turku, Finland \\ ${ }^{\mathrm{c}}$ Heart Centre, Turku University Central Hospital, Turku, Finland \\ ${ }^{\mathrm{d}}$ Division of Medicine, Turku University Central Hospital, Turku, Finland \\ e Department of Clinical Physiology, University of Tampere and Tampere University Hospital, Tampere, Finland \\ ${ }^{\mathrm{f}}$ Division of Cardiology, Heart and Lung Centre, Helsinki University Central Hospital, Helsinki, Finland \\ ${ }^{\mathrm{g}}$ National Heart, Lung, and Blood Institute's and Boston University's, Framingham Heart Study, Framingham, MA, USA
}

\begin{abstract}
Background: Scant data exist on incidence rates, correlates, and prognosis of electrocardiographic $\mathrm{P}$-wave abnormalities in the general population.

Methods: We recorded ECG and measured conventional cardiovascular risk factors in 5667 Finns who were followed up for incident atrial fibrillation (AF). We obtained repeat ECGs from 3089 individuals 11 years later.

Results: The incidence rates of prolonged P-wave duration, abnormal P terminal force (PTF), left Pwave axis deviation, and right $\mathrm{P}$-wave axis deviation were $16.0 \%, 7.4 \%, 3.4 \%$, and $2.2 \%$, respectively. Older age and higher BMI were associated with incident prolonged $\mathrm{P}$-wave duration and abnormal PTF $(P \leq 0.01)$. Higher blood pressure was associated with incident prolonged $\mathrm{P}$ wave duration and right $\mathrm{P}$-wave axis deviation $(P \leq 0.01)$. During follow-up, only prolonged $\mathrm{P}$ wave duration predicted AF (multivariable-adjusted hazard ratio, $1.38 ; P=0.001$ ).

Conclusions: Modifiable risk factors associate with P-wave abnormalities that are common and may represent intermediate steps of atrial cardiomyopathy on a pathway leading to AF.

(C) 2017 Elsevier Inc. All rights reserved.
\end{abstract}

Keywords: $\quad$ P-wave; Risk marker; Atrial fibrillation; Electrocardiography; Epidemiology

\section{Introduction}

Alterations in electrocardiographic P-wave morphology, e.g. prolonged $\mathrm{P}$-wave duration, $\mathrm{P}$ terminal force (PTF), and abnormal P-wave axis, may be indicative of atrial remodeling and of an underlying pathological process [1,2]. More importantly, alterations in P-wave morphology also seem to have clinical significance, as they have been shown to predict atrial fibrillation (AF) and ischemic stroke [3-6].

Aging, male sex, obesity, and hypertension have been shown to cross-sectionally correlate with prolonged P-wave duration and PTF [7-9]. However, cross-sectional data are not ideal for establishing causation, and longitudinal data on the incidence and correlates of abnormal P-wave indices are scant. A better

\footnotetext{
* Corresponding author at: Department of Health, National Institute for Health and Welfare, P.O. Box 57, 20521 Turku, Finland.

E-mail address: arolle@utu.fi (A.O. Lehtonen).
}

understanding of this domain could therefore elucidate the clinical factors mainly responsible for atrial electroanatomic remodeling, and help identify individuals at increased risk for incident AF and stroke. We therefore conducted a longitudinal study in a large, representative, nationwide population sample to determine 1) the prevalence and incidence rates of P-wave abnormalities in the general population; 2) the clinical factors associated with incident P-wave abnormalities; and 3) the distinct $\mathrm{P}$-wave abnormalities associated with incident AF.

\section{Material and methods}

Study sample

This study is based on the Health 2000 Survey, a nationwide epidemiological survey carried out in Finland between 2000 and 2001. The survey sample consisted of 
Table 1

Baseline characteristics by P-wave abnormality status.

\begin{tabular}{|c|c|c|c|c|c|c|c|c|c|c|c|}
\hline \multirow[t]{2}{*}{ Characteristic } & \multirow{2}{*}{$\begin{array}{l}\text { Study } \\
\text { sample }\end{array}$} & \multicolumn{3}{|c|}{ P-wave duration } & \multicolumn{3}{|c|}{$\mathrm{P}$ terminal force } & \multicolumn{4}{|l|}{$\mathrm{P}$-wave axis } \\
\hline & & $\leq 120 \mathrm{~ms}$ & $>120 \mathrm{~ms}$ & $P$ value & $>-4 \mathrm{mVms}$ & $\leq-4 \mathrm{mVms}$ & $P$ value & $0^{\circ}$ to $75^{\circ}$ & $<0^{\circ}$ & $>75^{\circ}$ & $P$ value \\
\hline$n$ & 5667 & 5107 & 560 & & 4807 & 860 & & 5114 & 291 & 262 & \\
\hline Age, years & $51.5(14.1)$ & $50.9(14.0)$ & $57.3(14.2)$ & $<0.001$ & $50.2(13.7)$ & $59.0(14.2)$ & $<0.001$ & $51.2(13.9)$ & $52.9(15.0)$ & $56.8(16.3)$ & $<0.001$ \\
\hline Men & $2577(45.5)$ & $2260(44.3)$ & $317(56.6)$ & $<0.001$ & $2177(45.3)$ & $400(46.5)$ & 0.50 & 2294 (44.9) & $144(49.5)$ & $139(53.1)$ & 0.01 \\
\hline $\begin{array}{c}\text { Systolic BP, } \\
\mathrm{mm} \mathrm{Hg}\end{array}$ & $134.1(20.8)$ & $133.4(20.6)$ & $140.1(21.6)$ & $<0.001$ & $132.4(20.2)$ & $143.5(21.8)$ & $<0.001$ & $134.0(20.7)$ & $134.7(21.0)$ & $135.2(21.9)$ & 0.69 \\
\hline $\begin{array}{c}\text { Diastolic BP, } \\
\mathrm{mm} \mathrm{Hg}\end{array}$ & $81.9(11.0)$ & $81.7(10.9)$ & $83.6(11.1)$ & $<0.001$ & $81.5(10.8)$ & $83.9(11.5)$ & $<0.001$ & $82.0(11.0)$ & $82.0(10.3)$ & $78.8(10.0)$ & $<0.001$ \\
\hline BMI, $\mathrm{kg} / \mathrm{m}^{2}$ & $26.9(4.6)$ & $26.7(4.6)$ & $28.1(4.7)$ & $<0.001$ & $26.8(4.6)$ & $27.6(4.6)$ & $<0.001$ & $27.0(4.6)$ & $27.2(4.2)$ & $24.6(4.6)$ & $<0.001$ \\
\hline $\begin{array}{c}\text { Non-HDL-C, } \\
\mathrm{mmol} / 1\end{array}$ & $4.6(1.1)$ & $4.6(1.2)$ & $4.7(1.1)$ & 0.007 & $4.6(1.2)$ & $4.8(1.1)$ & $<0.001$ & $4.6(1.1)$ & $4.6(1.1)$ & $4.4(1.2)$ & 0.006 \\
\hline Current smoking & $1249(22.0)$ & $1152(22.6)$ & $97(17.3)$ & 0.005 & $1074(22.3)$ & $175(20.4)$ & 0.19 & 1115 (21.8) & $59(20.3)$ & $75(28.6)$ & 0.03 \\
\hline Diabetes & $319(5.6)$ & $271(5.3)$ & $48(8.6)$ & 0.002 & 233 (4.9) & $86(10.0)$ & $<0.001$ & $278(5.4)$ & $22(7.6)$ & $19(7.3)$ & 0.16 \\
\hline Heart failure & 203 (3.6) & $157(3.1)$ & $46(8.2)$ & $<0.001$ & $140(2.9)$ & $63(7.3)$ & $<0.001$ & $172(3.4)$ & $9(3.1)$ & $22(8.4)$ & $<0.001$ \\
\hline CHD & 149 (2.6) & $107(2.1)$ & $42(7.5)$ & $<0.001$ & $102(2.1)$ & $47(5.5)$ & $<0.001$ & $121(2.4)$ & $11(3.8)$ & $17(6.5)$ & $<0.001$ \\
\hline COPD & $313(5.5)$ & $273(5.4)$ & $40(7.1)$ & 0.08 & $250(5.2)$ & $63(7.3)$ & 0.01 & $254(5.0)$ & $17(5.8)$ & $42(16.0)$ & $<0.001$ \\
\hline Heart rate/min & $63.1(10.6)$ & $63.3(10.5)$ & $61.4(11.3)$ & $<0.001$ & $62.6(10.4)$ & $65.6(11.5)$ & $<0.001$ & $63.1(10.6)$ & $61.2(9.1)$ & $64.6(11.1)$ & $<0.001$ \\
\hline $\begin{array}{r}\text { Chronotropic } \\
\text { medication }\end{array}$ & 809 (14.3) & $656(12.9)$ & $153(27.3)$ & $<0.001$ & $618(12.9)$ & $191(22.2)$ & $<0.001$ & 707 (13.8) & $57(19.6)$ & $45(17.2)$ & 0.009 \\
\hline
\end{tabular}

Values are means (standard deviations) for continuous data and numbers (percentages) for categorical data. BMI, body mass index; BP, blood pressure; CHD, coronary heart disease; COPD, chronic obstructive pulmonary disease; non-HDL-C, non-high-density lipoprotein cholesterol. $P$ value indicates the significance in difference (Wilcoxon rank sum test or Kruskal-Wallis test for continuous and chi-square test for categorical data) between the groups.

8028 individuals representative of the Finnish adult population aged $\geq 30$ years who were randomly drawn from the population register. The participation rate was $79 \%$ $(n=6354)$. A detailed description of the survey protocol and methods has been published previously [10].

The correlates of prevalent P-wave abnormalities and association between $\mathrm{P}$-wave abnormalities and incident $\mathrm{AF}$ were assessed in a sample where participants with one or more of the following exclusion factors were excluded: missing ECG $(n=55)$, previous diagnosis of $\mathrm{AF} /$ atrial flutter or AF/atrial flutter on the study ECG $(n=208)$, Wolf-Parkinson-White ECG pattern $(n=2)$, paced rhythm $(n=12)$, unmeasurable $\mathrm{P}$ waves $(n=110)$, or missing baseline covariates $(n=440)$. After exclusions, 5667 participants were included in these analyses.

A follow-up study of the Health 2000 Survey cohort, the Health 2011 Survey, was carried out in 2011-2012 using similar methods as the baseline survey. 3242 of the 5667 individuals included in the baseline analyses took part in the re-examination. After excluding participants with incident AF/atrial flutter $(n=140)$, Wolf-Parkinson-White ECG pattern $(n=0)$, paced rhythm $(n=12)$, or unmeasurable $\mathrm{P}$ waves at re-examination $(n=89), 3089(55 \%)$ participants had no missing baseline or follow-up data. After further excluding participants with prevalent P-wave abnormalities at baseline, 2831, 2710, 2948, and 2972 participants were included in the longitudinal analyses for correlates of incident prolonged P-wave duration, abnormal PTF, left $\mathrm{P}$-wave axis deviation, and right $\mathrm{P}$-wave axis deviation, respectively. We also assessed the reversal of P-wave abnormalities between baseline and follow-up in 258, 379, 141 and 117 participants with prolonged P-wave duration, abnormal PTF, left P-wave axis deviation, and right $\mathrm{P}$-wave axis deviation at baseline, respectively.

\section{Study flow}

Information on health, illnesses, medications and functional capacity were collected by centrally trained interviewers at the participants' homes during the baseline survey and at a local facility during the follow-up survey [10]. A physical examination was performed for each participant at a local health center by centrally trained doctors and nurses. Height, weight and body circumference were measured and fasting blood samples for serum lipids and glucose were taken from each participant. The study protocols were carried out according to the Declaration of Helsinki and were approved by the ethical committee of the hospital district of Helsinki and Uusimaa. All participants gave written informed consent.

\section{ECG recordings and definitions of $P$-wave abnormalities}

During the health examination, standard 12-lead ECGs were recorded in the resting supine position using standard procedures and a MAC 5000 recorder in 2000-2001 (Marquette Hellige, Freiburg, Germany, and Milwaukee, WI, USA) and a MAC5500 recorder (GE Healthcare, Freiburg, Germany) in 2011-2012. The electronic recordings were analyzed with the Magellan software (GE Healthcare, Milwaukee, WI, USA), and the measurements were manually checked, and if necessary, corrected by centrally trained nurses supervised by an experienced clinical physiologist.

Prolonged P-wave duration was determined as the maximum P-wave duration in any lead $>120 \mathrm{~ms}$ [5]. Abnormal PTF was determined as the product of the duration and the amplitude of the terminal portion of $\mathrm{P}$ wave $\leq-4 \mathrm{mVms}$ in lead V1 [11]. Left P-wave axis 
Table 2

Association between clinical variables and prevalent P-wave abnormalities.

\begin{tabular}{|c|c|c|c|c|}
\hline \multirow[b]{2}{*}{ Characteristic } & \multicolumn{4}{|c|}{ Odds ratios ( $95 \%$ confidence intervals) } \\
\hline & Prolonged P-wave duration & Abnormal $\mathrm{P}$ terminal force & P-wave axis $<0^{\circ}$ & $\mathrm{P}$-wave axis $>75^{\circ}$ \\
\hline Age, 10 years & $1.26(1.16-1.36)^{* * *}$ & $1.41(1.32-1.50)^{* * *}$ & $1.09(0.98-1.21)$ & $1.36(1.22-1.51)^{* * *}$ \\
\hline Men & $1.79(1.48-2.15)^{* * *}$ & $1.09(0.93-1.27)$ & $1.24(0.97-1.59)$ & $1.54(1.18-2.01)^{* *}$ \\
\hline Systolic BP, $10 \mathrm{~mm} \mathrm{Hg}$ & $1.02(0.98-1.08)$ & $1.14(1.09-1.18)^{* * *}$ & $0.97(0.90-1.04)$ & $0.99(0.93-1.07)$ \\
\hline BMI, $5 \mathrm{~kg} / \mathrm{m}^{2}$ & $1.23(1.11-1.36)^{* * *}$ & $1.02(0.94-1.12)$ & $1.02(0.89-1.18)$ & $0.45(0.37-0.54)^{* * *}$ \\
\hline Non-HDL-C, mmol/1 & $0.98(0.91-1.07)$ & $1.01(0.95-1.09)$ & $0.97(0.87-1.08)$ & $0.86(0.76-0.97)^{*}$ \\
\hline Current smoking & $0.87(0.69-1.11)$ & $1.27(1.05-1.54)^{*}$ & $0.94(0.70-1.28)$ & $1.41(1.05-1.91)^{*}$ \\
\hline Diabetes & $0.90(0.64-1.27)$ & $1.27(0.96-1.68)$ & $1.26(0.78-2.02)$ & $1.18(0.70-2.00)$ \\
\hline CHD & $1.52(1.00-2.32)$ & $1.43(0.96-2.14)$ & $1.14(0.57-2.29)$ & $1.33(0.70-2.52)$ \\
\hline Heart failure & $1.28(0.87-1.89)$ & $1.22(0.86-1.72)$ & $0.62(0.30-1.28)$ & $1.97(1.13-3.43)^{*}$ \\
\hline COPD & $0.81(0.56-1.17)$ & $0.71(0.52-0.97)^{*}$ & $1.03(0.61-1.74)$ & $1.81(1.21-2.71)^{* *}$ \\
\hline Chronotropic medication & $1.58(1.24-2.01)^{* * *}$ & $0.98(0.79-1.22)$ & $1.44(1.02-2.03)^{*}$ & $0.94(0.62-1.40)$ \\
\hline
\end{tabular}

BMI, body mass index; BP, blood pressure; CHD, coronary heart disease; COPD, chronic obstructive pulmonary disease; non-HDL-C, non-high-density lipoprotein cholesterol. Each of the four models included all the listed characteristics as covariates. Odds ratios for P-wave axis were computed using $0^{\circ}$ to $75^{\circ}$ as reference.

$* \mathrm{P}<0.05$

$* * \mathrm{P}<0.01$;

$* * * \mathrm{P}<0.001$;

deviation was defined as $\mathrm{P}$-wave axis $<0^{\circ}$ and right $\mathrm{P}$-wave axis deviation as $\mathrm{P}$-wave axis $>75^{\circ}[12]$.

\section{Covariates}

Blood pressure was measured with a standard mercury manometer from the right arm after a 10-min rest. The means of two measurements performed at a two-minute interval were used to determine blood pressure. Diabetes mellitus was defined as fasting serum glucose level of $7.0 \mathrm{mmol} / \mathrm{L}$ or greater, or a history of the use of oral hypoglycemic agents or insulin therapy. Current smoking was defined as daily use of tobacco products. Serum non-high-density lipoprotein (non-HDL) cholesterol concentration was calculated as total cholesterol minus HDL-cholesterol. Chronic obstructive pulmonary disease (COPD) was defined as having been diagnosed with COPD by a physician and receiving medication for COPD; or having a spirometry result indicative of COPD according to the Global Initiative for Chronic Obstructive Lung Disease criteria [13]. Use of chronotropic medication (beta-blockers, digitalis, calcium-channel blockers, and antiarrhythmic agents) was determined based on the participant's self-reported use of medication prescriptions in the health interview.

\section{Prevalent cardiac disease and follow-up for $A F$}

Information on prevalent and incident cardiovascular disease was obtained from the National Hospital Discharge register. This register contains information on all periods of inpatient (since 1967) and outpatient (since 1998) care in all Finnish secondary- and tertiary-care hospitals. Diagnoses are registered in this register by the treating physicians with codes defined in the 10th revision of the International Classification of Diseases (ICD-10). In addition, data on causes of death, drug purchases, and entitlements to special reimbursements due to chronic diseases was gathered from the Causes of Death and Drug Reimbursement registers. All aforementioned registers are nationwide with a full coverage for all participants who continued to live in Finland.
Prevalent coronary heart disease was determined as previous hospitalization with ICD-10 codes I20-I22 and/or percutaneous coronary intervention or coronary artery bypass surgery. Prevalent heart failure was determined as previous hospitalization with any of the ICD-10 codes I50, I110, I130, I132; or if a participant had purchased furosemide on at least three occasions or had obtained the right for reimbursement for medication costs for heart failure. The coronary heart disease and heart failure diagnoses of the previously mentioned registers have been previously validated $[14,15]$. Incident AF was defined as the ICD-10 code I48 in the National Hospital Discharge or Causes of Death registers during follow-up or AF in the Health 2011 Survey follow-up ECG. Follow-up for incident AF ended on December 31, 2013.

\section{Statistical analyses}

We assessed the differences in baseline characteristics between individuals with and without $\mathrm{P}$-wave abnormalities using the chi-squared, the Wilcoxon rank sum, and the Kruskal-Wallis tests. We examined the cross-sectional and longitudinal associations between baseline characteristics and $\mathrm{P}$-wave abnormalities using logistic regression. We included baseline age, sex, systolic blood pressure, BMI, non-HDL-cholesterol, smoking, diabetes, use of chronotropic medication, COPD, prevalent heart failure, and prevalent coronary heart disease in the models as independent variables. We included these variables in the models due to their significance as cardiovascular risk factors, or due to their potential impact on the P-wave abnormalities. We used Cox proportional hazards regression model to evaluate the association of $\mathrm{P}$-wave abnormalities with incident AF. We adjusted these models for baseline age, sex, smoking, systolic blood pressure, non-HDL-cholesterol, BMI, diabetes, coronary heart disease, heart failure, and heart rate. We confirmed the proportional hazards assumption with the Kolmogorov-type supremum test. We performed a sensitivity analysis with abnormal PTF defined as $\leq-6 \mathrm{mVms}$ as in 

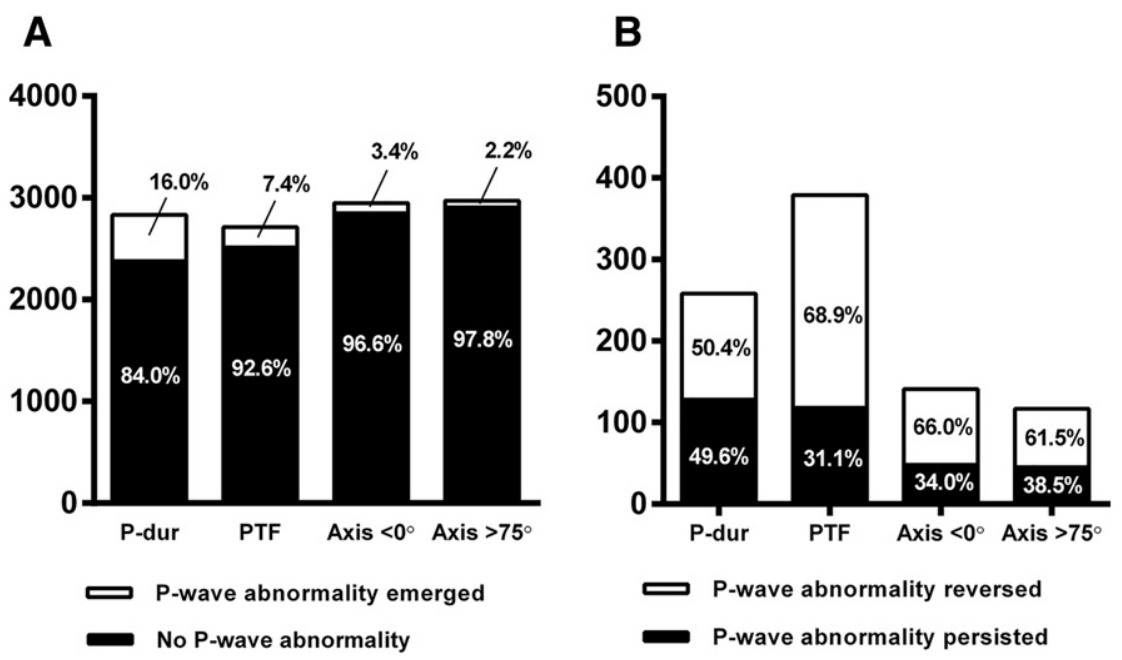

Fig. 1. Longitudinal changes in P-wave abnormalities during 11-year follow-up. Panel A: Incidence of P-wave abnormalities in individuals without P-wave abnormalities at baseline. Panel B: Reversal of P-wave abnormalities in individuals with P-wave abnormalities at baseline. P-dur, P-wave duration; PTF, P terminal force.

a previous publication [16]. We used Kaplan-Meier curves to evaluate the incidence of $\mathrm{AF}$ in participants with and without prolonged P-wave duration. All statistical analyses were performed using SAS 9.4 (SAS Institute Inc., Cary, NC, USA). Two-tailed $P<0.05$ was considered statistically significant.

\section{Results}

\section{Study sample}

The baseline characteristics of the whole study sample are shown in Table 1. The prevalence rates of prolonged P-wave duration, abnormal PTF, left P-wave axis deviation, and right P-wave axis deviation were 9.9\%, 15.2\%, 5.1\%, and $4.6 \%$ at baseline, respectively. Participants with P-wave abnormal- ities were older and had a greater prevalence of heart failure, coronary heart disease, and chronotropic medication use than those with normal P-waves.

\section{Cross-sectional determinants of $P$-wave abnormalities}

In multivariable-adjusted logistic regression analyses, participants with prolonged P-wave duration, abnormal PTF, and right $\mathrm{P}$-wave axis deviation were more likely to be older (Table 2). Participants with prolonged P-wave duration were more likely to be male, have higher BMI, and use chronotropic medication. Participants with abnormal PTF were more likely to have higher systolic blood pressure and smoke, but less likely to have COPD. Participants with right P-wave axis deviation were more likely to be male, smoke, and have COPD, heart failure, lower BMI, and lower non-HDL-cholesterol.

Table 3

Association between clinical variables and incident $\mathrm{P}$-wave abnormalities.

\begin{tabular}{lllll}
\hline Characteristic & \multicolumn{4}{l}{ Odds ratios $(95 \%$ confidence intervals $)$} \\
\cline { 2 - 5 } & $\begin{array}{l}\text { Prolonged P-wave duration } \\
(n=453 \text { of } 2831 \text { analyzed })\end{array}$ & $\begin{array}{l}\text { Abnormal P terminal force } \\
(n=200 \text { of } 2710 \text { analyzed })\end{array}$ & $\begin{array}{l}\text { P-wave axis }<0^{\circ} \\
(\mathrm{n}=99 \text { of } 2948 \text { analyzed })\end{array}$ & $\begin{array}{l}\text { P-wave axis }>75^{\circ} \\
(\mathrm{n}=66 \text { of } 2972 \text { analyzed })\end{array}$ \\
\hline Age, 10 years & $1.29(1.16-1.44)^{* * *}$ & $1.53(1.31-1.79)^{* * *}$ & $1.01(0.82-1.25)$ & $1.12(0.86-1.45)$ \\
Men & $2.14(1.72-2.66)^{* * *}$ & $1.08(0.80-1.46)$ & $1.19(0.79-1.79)$ & $1.06(0.64-1.77)$ \\
Systolic BP, $10 \mathrm{~mm} \mathrm{Hg}$ & $1.11(1.04-1.18)^{* *}$ & $1.01(0.93-1.11)$ & $1.01(0.89-1.14)$ & $1.23(1.08-1.40)^{* *}$ \\
BMI, $5 \mathrm{~kg} / \mathrm{m}^{2}$ & $1.53(1.35-1.74)^{* * *}$ & $1.26(1.06-1.50)^{* *}$ & $1.01(0.79-1.29)$ & $0.49(0.34-0.71)^{* * *}$ \\
Non-HDL-C, mmol/1 & $0.98(0.88-1.08)$ & $1.07(0.93-1.22)$ & $1.10(0.91-1.32)$ & $0.88(0.69-1.13)$ \\
Current smoking & $0.83(0.62-1.11)$ & $1.14(0.76-1.71)$ & $0.73(0.42-1.28)$ & $1.94(1.12-3.36)^{*}$ \\
Diabetes & $0.86(0.49-1.51)$ & $1.21(0.55-2.62)$ & $0.95(0.32-2.88)$ & $0.19(0.01-2.97)$ \\
CHD & $0.93(0.40-2.17)$ & $3.21(1.35-7.59)^{* *}$ & $1.28(0.31-5.25)$ & $0.56(0.07-4.48)$ \\
Heart failure & $1.03(0.45-2.36)$ & $0.59(0.17-2.03)$ & $2.63(0.81-8.55)$ & $5.51(1.44-21.09)^{*}$ \\
COPD & $1.32(0.71-2.45)$ & $0.42(0.13-1.39)$ & $0.95(0.26-3.47)$ & $4.34(1.83-10.33)^{* * *}$ \\
Chronotropic medication & $1.13(0.78-1.63)$ & $1.18(0.73-1.91)$ & $1.50(0.79-2.86)$ & $1.21(0.53-2.79)$ \\
\hline
\end{tabular}

BP, blood pressure; BMI, body mass index; CHD, coronary heart disease; COPD, chronic obstructive pulmonary disease; non-HDL-C, non-high-density lipoprotein cholesterol. Each of the four models included all the listed characteristics as covariates.

$$
\begin{aligned}
& * \mathrm{P}<0.05 ; \\
& * * \mathrm{P}<0.01 ; \\
& * * * \quad \mathrm{P}<0.001 ;
\end{aligned}
$$


Table 4

Association between P-wave abnormalities and incident atrial fibrillation.

\begin{tabular}{|c|c|c|c|c|c|c|c|}
\hline \multirow[b]{2}{*}{$\mathrm{P}$-wave characteristic } & \multirow[b]{2}{*}{$n$} & \multirow[b]{2}{*}{ AF events $(\%)$} & \multirow[b]{2}{*}{ AF events per 1000 person-years } & \multicolumn{4}{|l|}{ Hazard ratio } \\
\hline & & & & Crude (95\% CI) & $P$ value & Adjusted $^{\dagger}(95 \% \mathrm{CI})$ & $P$ value \\
\hline \multicolumn{8}{|l|}{ P-wave duration } \\
\hline$\leq 120 \mathrm{~ms}$ & 5107 & $337(6.6)$ & 5.5 & 1.00 (reference) & & 1.00 (reference) & \\
\hline$>120 \mathrm{~ms}$ & 560 & $86(15.4)$ & 13.7 & $2.52(1.99-3.20)$ & $<0.001$ & $1.38(1.08-1.76)$ & 0.001 \\
\hline \multicolumn{8}{|l|}{ P terminal force } \\
\hline$>-4 \mathrm{mVms}$ & 4807 & $331(6.9)$ & 5.7 & 1.00 (reference) & & 1.00 (reference) & \\
\hline$\leq-4 \mathrm{mVms}$ & 860 & $92(10.7)$ & 9.7 & $1.72(1.37-2.17)$ & $<0.001$ & $0.95(0.75-1.21)$ & 0.68 \\
\hline \multicolumn{8}{|l|}{$\mathrm{P}$-wave axis } \\
\hline $0^{\circ}-75^{\circ}$ & 5114 & $363(7.1)$ & 5.9 & 1.00 (reference) & & 1.00 (reference) & \\
\hline$<0^{\circ}$ & 291 & $30(10.3)$ & 8.8 & $1.50(1.03-2.18)$ & 0.03 & $1.16(0.80-1.69)$ & 0.43 \\
\hline$>75^{\circ}$ & 262 & $30(11.5)$ & 10.6 & $1.81(1.25-2.63)$ & 0.002 & $1.31(0.90-1.92)$ & 0.16 \\
\hline
\end{tabular}

$\mathrm{AF}$, atrial fibrillation; CI, confidence interval. ${ }^{\dagger}$ Adjusted for baseline age, sex, systolic blood pressure, BMI, non-HDL-cholesterol, current smoking, diabetes, prevalent heart failure, prevalent coronary heart disease and heart rate.

\section{Longitudinal changes and determinants of change in $P$-wave abnormalities}

Changes in P-wave abnormality statuses during follow-up are shown in Fig. 1. The incidence rates of prolonged P-wave duration, abnormal PTF, left P-wave axis deviation, and right $\mathrm{P}$-wave axis deviation after an 11-year follow-up were $16.0 \%, 7.4 \%, 3.4 \%$ and $2.2 \%$, respectively. The reversal of prolonged $\mathrm{P}$-wave duration, abnormal PTF, left P-wave axis deviation, and right $\mathrm{P}$-wave axis deviation occurred respectively in $50.4 \%, 68.9 \%, 66.0 \%$, and $61.5 \%$ of the participants with prevalent $\mathrm{P}$-wave abnormalities at baseline. The association of baseline characteristics with incident $\mathrm{P}$-wave abnormalities is shown in Table 3. Older age, male sex, higher systolic blood pressure, and higher BMI were associated with incident prolonged $\mathrm{P}$-wave duration. Older age, higher BMI, and prevalent coronary heart disease were related to incident abnormal PTF. Higher systolic blood pressure, lower BMI, smoking, prevalent heart failure, and prevalent COPD were associated with incident right $\mathrm{P}$-wave axis deviation.

To assess the possibility of selection bias in the longitudinal analyses; we compared the baseline characteristics of participants who were re-examined to those who did not attend re-examination. Participants who were not re-examined were older and more likely to be men ( $P<0.001$ for both, data not shown). In addition, these individuals had higher blood pressure, higher BMI, higher non-HDL-cholesterol, higher heart rate, and greater prevalence rates of diabetes, coronary heart disease, COPD, heart failure and chronotropic medication use than those who attended re-examination ( $P<0.001$ for all, data not shown). In addition, participants who were not re-examined had significantly greater baseline P-wave duration (104.7 ms vs. $103.9 \mathrm{~ms} ; \quad P=0.03)$ and PTF $(-2.3 \mathrm{mVms}$ vs. $2.0 \mathrm{mVms}, P<0.001)$ than those who were re-examined.

\section{Association of P-wave abnormalities with incident AF}

During a mean follow-up of $11.9 \pm 2.9$ years, 423 participants had $\geq 1$ episode of AF. The unadjusted and multivariable-adjusted hazard ratios (HR) and 95\% confidence intervals (CI) for incident AF are presented in Table 4. In the unadjusted Cox models, the rates of incident AF were higher among participants with baseline prolonged P-wave duration (HR, 2.52; 95\% CI, 1.99-3.20), abnormal PTF (HR, 1.72; 95\% CI, 1.37-2.17), left P-wave axis deviation (HR, 1.50; 95\% CI, 1.03-2.18), and right $\mathrm{P}$-wave axis deviation (HR, 1.81; 95\% CI, 1.25-2.63). However, in the multivariable-adjusted models, only prolonged P-wave duration was a significant predictor of AF: (HR, 1.38; 95\% CI, 1.08-1.76). This association became even stronger (HR, 1.75 ; 95\% CI, 1.27-2.41) when individuals on chronotropic medication $(\mathrm{n}=809)$ were excluded. The cumulative risk of $\mathrm{AF}$ in individuals with and without prolonged $\mathrm{P}$-wave duration is illustrated in Fig. 2. Abnormal PTF and P-wave axis were not associated with incident AF in the multivariable-adjusted models in the overall sample and in participants not on chronotropic medication $(P \geq 0.16$ for all). We also performed a sensitivity analysis with PTF defined as $\leq-6 \mathrm{mVms}$ (data not shown) and found no association between this marker and incident $\operatorname{AF}(P=0.23)$. However, when participants on chronotropic medication were excluded, the association between abnormal PTF (defined as $\leq-6 \mathrm{mVms}$ ) and AF became significant (HR, $1.79 ; 95 \%$ CI, $1.14-2.82 ; P=0.01)$.

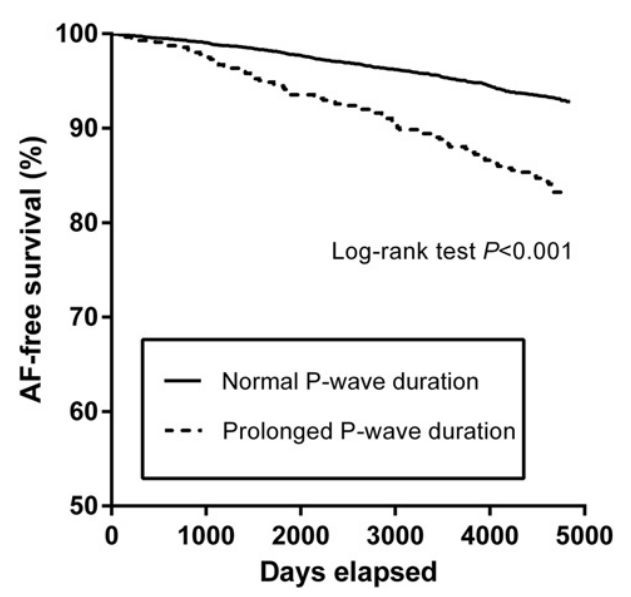

Fig. 2. Kaplan-Meier curves for incident atrial fibrillation in participants with normal and prolonged $(>120 \mathrm{~ms})$ P-wave duration. AF, atrial fibrillation. 


\section{Discussion}

Our results demonstrate that the prevalence and incidence rates of $\mathrm{P}$-wave abnormalities are relatively high in the general population. In addition, our results demonstrate high reversal rates of $\mathrm{P}$-wave abnormalities during follow-up. To the best of our knowledge, this study is the first to describe the long-term changes in electrocardiographic atrial measures. Older age, systolic blood pressure and BMI, in particular, were associated with prevalent and incident P-wave abnormalities. Furthermore, we confirmed in an unselected, nationwide, population sample previous findings suggestive of an association between prolonged P-wave duration and AF. In addition, abnormal PTF defined as $\leq-$ $6 \mathrm{mVms}$, but not as $\leq-4 \mathrm{mVms}$, predicted AF in participants not on chronotropic medication. Abnormal P-wave axis was not related to incident AF in our sample.

\section{Prolonged P-wave duration}

We observed that prolonged P-wave duration is a common phenomenon in the general population. At baseline, 9.9\% of individuals in our sample had prolonged $\mathrm{P}$-wave duration, and it emerged in $16.0 \%$ of participants during follow-up. In agreement with previous studies [7-9], older age, male sex and higher BMI were cross-sectionally associated with prolonged P-wave duration in our study. The same variables, plus systolic blood pressure, were also independently related to incident prolonged $\mathrm{P}$-wave duration. In view of these findings, especially BMI and hypertension seem to contribute causally to the electroanatomic remodeling of the atria.

In our study, prolonged $\mathrm{P}$ wave duration was predictive of AF. In addition, this association became even stronger when participants on chronotropic medication were excluded using a similar approach as in a previous publication [5]. These results are in accordance with findings from previous studies performed with patient [17] and population [4,5] cohorts that have shown an association between prolonged P-wave duration and incident AF.

\section{Abnormal PTF}

The prevalence of abnormal PTF in our study was $15.2 \%$. This is slightly higher than in previous studies with manual or automatized ECG measurements, in which the observed prevalence rates were $7.5 \%$, and $10.1 \%$ respectively $[6,16]$. Previous cross-sectional works have identified advancing age, BMI and hypertension as correlates of abnormal PTF $[7,8]$. In addition to an association with older age and higher systolic blood pressure, we found that abnormal PTF was positively related to smoking and inversely related to COPD cross-sectionally. This inverse relationship with COPD is an expected finding and underlines that lung disorders need to be accounted for in analyses concerning PTF. However, in our longitudinal analyses, older age, higher BMI and prevalent coronary heart disease, were associated with incident abnormal PTF. The observed associations between higher BMI, and both abnormal PTF and prolonged P-wave duration, strongly suggest a link between obesity and development of atrial cardiomyopathy.
In contrast to previous large-scale epidemiological studies $[4,5]$, abnormal PTF was not associated with incident AF in our study. However, this association became significant in participants not on chronotropic medication when abnormal PTF was defined as $\leq-6 \mathrm{mVms}$ instead of $\leq-4 \mathrm{mVms}$ as in a previous publication [16]. In addition to supporting a limited association between incident AF and abnormal PTF, our results also raise the need to study the optimal definition of abnormal PTF.

\section{P-wave axis deviation}

In accord with our results, previous studies have observed that individuals with abnormal $\mathrm{P}$-wave axis are elderly with tendency for low BMI and high prevalence of COPD [3,12]. Our study further clarifies that these associations are largely driven by right $\mathrm{P}$-wave axis deviation instead of left $\mathrm{P}$-wave axis deviation. In our study, higher systolic blood pressure, lower BMI and prevalent COPD were associated with the incident right $\mathrm{P}$-wave axis deviation. The strong association between COPD and right $\mathrm{P}$-wave axis deviation is not surprising as lung disorders affect the orientation of the heart in the thoracic cavity and, thus, cause a rightward shift to the P-wave axis [18]. On the other hand, central obesity may affect the diaphragm causing a leftward shift to the P-wave axis, which explains the association between lower BMI and rightward P-wave axis [19].

In contrast to a previous study [3], abnormal P-wave axis was not associated with incident AF in our study which may be due to differences in study samples. In the study by Rangel et al., the participants were older with a higher prevalence of abnormal P-wave axis and a higher incidence of AF than our study. Furthermore, participants with abnormal P-wave axis in our study had significantly higher prevalence rates of heart failure and coronary heart disease than those in the study by Rangel et al. which could explain the observed lack of association between abnormal P-wave axis and incident AF. In a previous study, we observed no association between abnormal P-wave axis and incident coronary heart disease supporting a limited prognostic significance of abnormal P-wave axis in the general population [20].

\section{Reversal of $P$-wave abnormalities}

It is important to note that over half of the baseline P-wave abnormalities had reversed to normal at re-examination, which suggests considerable lability in P-wave morphology. However, this may also in large part reflect regression to the mean. Furthermore, our results may overestimate the reversal rates of $\mathrm{P}$-wave abnormalities to some extent because individuals with the most severe forms of P-wave abnormalities were less likely to attend the re-examination. Unfortunately, the low number of individuals with prevalent $\mathrm{P}$-wave abnormalities that reversed to normal during follow-up prevented us from performing formal analyses on the possible correlates of this phenomenon. In any case, this finding warrants further research in even larger population cohorts. 


\section{Context to current literature}

P-wave abnormalities have been suggested as reflections of electroanatomic remodeling of the atria, and thereby a potential substrate for AF $[2,3,5]$. Consequently, our results underscore the potential usefulness of P-wave abnormalities as intermediate biomarkers of atrial cardiomyopathy on a pathway leading to AF. We observed that several risk factors that associate with AF and ischemic stroke, such as obesity and hypertension, also seem to be associated with the incidence of prolonged P-wave duration and abnormal PTF. Of note, both obesity and hypertension are modifiable risk factors and treating them reduces the risk of incident $\mathrm{AF}$ $[21,22]$. Our study suggests that changes in P-wave abnormalities could possibly be used for the evaluation of treatment success and estimating risk of incident AF. In addition, given the usefulness of $\mathrm{P}$-wave abnormalities in ischemic stroke prediction in individuals without evidence of $\mathrm{AF}$, future studies comparing anticoagulation versus antiplatelet therapy in individuals with ECG markers of atrial cardiomyopathy might be warranted [23]. In recent years, partial and advanced interatrial blocks have also gained wide interest in this setting and proved to have considerable clinical relevance [24-27]. Advanced interatrial block in particular, defined as prolonged $\mathrm{P}$-wave duration with biphasic waveform in inferior leads, has been shown to strongly predict $\mathrm{AF}$ and stroke in the general population $[28,29]$. Unfortunately, we were underpowered to study the correlates and prognosis of advanced interatrial block in our study sample because of the low number of individuals affected with advanced interatrial block.

\section{Limitations}

Our study has several limitations. First, although our baseline study sample was relatively large, approximately half of the participants were not re-examined due to death and other causes, which lowers the representativeness of the follow-up sample. However, this limitation affects only the results on incidence and reversal of P-wave abnormalities, and not results concerning incident $\mathrm{AF}$ that were based on nationwide register data. Second, P-wave measurements were performed on only two visits which may not be enough to describe the full natural history of P-wave abnormalities. However, our study is the first to describe the long-term changes in P-wave abnormalities. Third, we are not yet able to assess how the long-term changes of P-wave abnormalities affect the risk of incident AF. Fourth, the data on prevalent and incident AF were primarily based on register information from secondary and tertiary care. As a consequence, it is likely that some AF paroxysms were only diagnosed in primary care or were left undiagnosed and were thus not accounted for in our analyses. Fifth, our results may not be generalizable to other ethnicities. Finally, echocardiographic data on atrial measures were not available for this study. However, electrocardiographic and echocardiographic variables measure different aspects of the heart's physiology and therefore complement one another [17].

\section{Conclusions}

Our results contribute to the existing literature on P-wave abnormalities and clarify the role of P-wave abnormalities as intermediate risk factors for AF. We conclude that P-wave abnormalities are frequent findings in the general population. Our results also highlight high reversal rates of P-wave abnormalities during follow-up - a finding that requires confirmation in other populations. Modifiable risk factors that have been previously shown to associate with incident $\mathrm{AF}$ and stroke, such as obesity and hypertension, also seem to be associated with the incidence of prolonged P-wave duration and abnormal PTF. P-wave abnormalities, and especially prolonged P-wave duration, may represent intermediate steps of atrial cardiomyopathy on a pathway leading to $\mathrm{AF}$ and can be used to estimate future risk of $\mathrm{AF}$.

\section{Conflicts of interest}

None.

\section{Acknowledgements}

The authors thank the investigators, staff and participants of the Health 2000 and the Health 2011 surveys for their contributions. Arttu Lehtonen was supported by grants from the Uulo Arhio Fund, the Ida Montin Foundation, and the Betania Foundation.

\section{References}

[1] Magnani JW, Williamson MA, Ellinor PT, Monahan KM, Benjamin EJ $P$ wave indices: current status and future directions in epidemiology, clinical, and research applications. Circ Arrhythm Electrophysiol 2009;2(1):72-9.

[2] Platonov PG. P-wave morphology: underlying mechanisms and clinical implications. Ann Noninvasive Electrocardiol 2012;17(3):161-9.

[3] Rangel MO, O'Neal WT, Soliman EZ. Usefulness of the electrocardiographic P-wave axis as a predictor of atrial fibrillation. Cardiol 2016;117(1):100-4.

[4] Soliman EZ, Prineas RJ, Case LD, Zhang Z, Goff DC. Ethnic distribution of ECG predictors of atrial fibrillation and its impact on understanding the ethnic distribution of ischemic stroke in the Atherosclerosis Risk in Communities (ARIC) study. Stroke 2009;40(4):1204-11.

[5] Magnani JW, Zhu L, Lopez F, Pencina MJ, Agarwal SK, Soliman EZ, et al. P-wave indices and atrial fibrillation: cross-cohort assessments from the Framingham Heart Study (FHS) and Atherosclerosis Risk in Communities (ARIC) study. Am Heart J 2015;169(1) [53,61.e1].

[6] Kamel H, O’Neal WT, Okin PM, Loehr LR, Alonso A, Soliman EZ. Electrocardiographic left atrial abnormality and stroke subtype in ARIC. Ann Neurol 2015;78(5):670-8

[7] Magnani JW, Lopez FL, Soliman EZ, Maclehose RF, Crow RS, Alonso A. $\mathrm{P}$ wave indices, obesity, and the metabolic syndrome: the Atherosclerosis Risk in Communities study. Obes Sci Pract 2012;20(3):666-72.

[8] Soliman EZ, Alonso A, Misialek JR, Jain A, Watson KE, Lloyd-Jones $\mathrm{DM}$, et al. Reference ranges of PR duration and P-wave indices in individuals free of cardiovascular disease: the Multi-Ethnic Study of Atherosclerosis (MESA). J Electrocardiol 2013;46(6):702-6.

[9] Vaidean GD, Manczuk M, Magnani JW. Atrial electrocardiography in obesity and hypertension: clinical insights from the Polish-Norwegian Study (PONS). Obes Sci Pract 2016;24(12):2608-14.

[10] Heistaro S. Methdology report. The health 2000 survey. Helsinki, Finland: National Public Health Institute; 2008. [http://urn.fi/URN: NBN:fi-fe201204193320].

[11] Morris JJ, Estes EH, Whalen RE, Thompson HK, McIntosh HD. PWave analysis in valvular heart disease. Circulation 1964;29:242-52. 
[12] Li Y, Shah AJ, Soliman EZ. Effect of electrocardiographic P-wave axis on mortality. Cardiol 2014;113(2):372-6.

[13] World Health Organization, National Heart, Lung and Blood Institute. Global strategy for the diagnosis, management, and prevention of chronic obstructive pulmonary disease. Global initiative for chronic obstructive lung disease (GOLD); 2016.

[14] Pajunen P, Koukkunen H, Ketonen M, Jerkkola T, Immonen-Räihä P, Kärjä-Koskenkari $\mathrm{P}$, et al. The validity of the Finnish hospital discharge register and causes of death register data on coronary heart disease. Cardiovasc Prev Rehabil 2005;12(2):132-7.

[15] Mähönen M, Jula A, Harald K, Antikainen R, Tuomilehto J, Zeller T, et al. The validity of heart failure diagnoses obtained from administrative registers. Prev Cardiol 2013;20(2):254-9.

[16] Eranti A, Aro AL, Kerola T, Anttonen O, Rissanen HA, Tikkanen JT, et al. Prevalence and prognostic significance of abnormal $\mathrm{P}$ terminal force in lead V1 of the ECG in the general population. Circ Arrhythm Electrophysiol 2014;7(6):1116-21.

[17] Nielsen JB, Kühl JT, Pietersen A, Graff C, Lind B, Struijk JJ, et al. Pwave duration and the risk of atrial fibrillation: results from the Copenhagen ECG study. Heart Rhythm 2015;12(9):1887-95.

[18] Surawicz B. Atrial abnormalities. In: Surawicz B, Knilans T, editors. Chou's electrocardiography in clinical practice: adult and pediatric. 6th ed. Philadelphia: Saunders Elsevier; 2008. p. 33-6.

[19] Fraley MA, Birchem JA, Senkottaiyan N, Alpert MA. Obesity and the electrocardiogram. Obes Rev 2005;6(4):275-81.

[20] Lehtonen AO, Puukka P, Varis J, Porthan K, Tikkanen JT, Nieminen MS, et al. Prevalence and prognosis of ECG abnormalities in normotensive and hypertensive individuals. J Hypertens 2016;34(5):959-66.
[21] Emdin CA, Callender T, Cao J, Rahimi K. Effect of antihypertensive agents on risk of atrial fibrillation: a meta-analysis of large-scale randomized trials. Europace 2015;17(5):701-10.

[22] Jamaly S, Carlsson L, Peltonen M, Jacobson P, Sjostrom L, Karason K. Bariatric surgery and the risk of new-onset atrial fibrillation in Swedish obese subjects. J Am Coll Cardiol 2016;68(23):2497-504.

[23] Kamel H, Okin PM, Elkind MSV, Iadecola C. Atrial fibrillation and mechanisms of stroke: time for a new model. Stroke 2016:47(3):895-900.

[24] Bayés de Luna A, Platonov P, Cosio FG, Cygankiewicz I, Pastore C, Baranowski R, et al. Interatrial blocks. A separate entity from left atrial enlargement: a consensus report. J Electrocardiol 2012;45(5):445-51.

[25] Maheshwari A, Norby FL, Soliman EZ, Alraies MC, Adabag S, O'Neal WT, et al. Relation of prolonged P-wave duration to risk of sudden cardiac death in the general population (from the Atherosclerosis Risk in Communities study). Cardiol 2017;119(9):1302-6.

[26] Wu JT, Wang SL, Chu YJ, Long DY, Dong JZ, Fan XW, et al. Usefulness of a combination of interatrial block and a high CHADS2 score to predict new onset atrial fibrillation. Int Heart J 2016;57(5):580-5.

[27] Wu JT, Wang SL, Chu YJ, Long DY, Dong JZ, Fan XW, et al. CHADS2 and CHA2DS2-VASc scores predict the risk of ischemic stroke outcome in patients with interatrial block without atrial fibrillation. J Atheroscler Thromb 2017;24(2):176-84.

[28] O'Neal WT, Zhang Z, Loehr LR, Chen LY, Alonso A, Soliman EZ. Electrocardiographic advanced interatrial block and atrial fibrillation risk in the general population. Cardiol 2016;117(11):1755-9.

[29] O'Neal WT, Kamel H, Zhang ZM, Chen LY, Alonso A, Soliman EZ. Advanced interatrial block and ischemic stroke: the atherosclerosis risk in communities study. Neurology 2016;87(4):352-6. 